\title{
Breastfeeding and the Risk of Childhood Leukemia: A Meta-Analysis
}

\author{
Marilyn L. Kwan, PhD \\ Patricia A. Buffler, PhD, \\ $\mathrm{MPH}^{\mathrm{a}}$ \\ BARBARA ABrams, DrPH ${ }^{a}$ \\ Vincent A. Kiley, MD
}

\section{SYNOPSIS}

Objectives. The authors used a meta-analytic technique to (1) quantify the evidence of an association between duration of breastfeeding and risk of childhood acute lymphoblastic leukemia (ALL) or acute myeloblastic leukemia (AML), (2) assess the influence of socioeconomic status (SES) on any such associations, and (3) discuss the implications of these findings for the evaluation of whether breastfeeding reduces the risk of childhood leukemia.

Methods. A fixed effects model was employed to systematically combine the results of 14 case-control studies addressing the effect of short-term ( $\leq 6$ months) and long-term ( $>6$ months) breastfeeding on the risk of childhood ALL and/or AML. Subgroup analyses of studies that did and did not adjust for SES were also performed.

Results. A significant, negative association was observed between long-term breastfeeding and both ALL risk (odds ratio $[O R]=0.76$; $95 \%$ confidence interval $[\mathrm{Cl}] 0.68,0.84)$ and $A M L$ risk $(O R=0.85$; $95 \% \mathrm{Cl} 0.73,0.98)$. Short-term breastfeeding was similarly protective for ALL and AML. Results for studies that adjusted and did not adjust for SES were not significantly different from the results for the 14 studies combined.

Conclusions. This meta-analysis showed that both short-term and long-term breastfeeding reduced the risk of childhood ALL and $A M L$, suggesting that the protective effect of breastfeeding might not be limited to ALL as earlier hypothesized. Potential bias introduced by different participation rates for case and control samples that differed in SES can be minimized by implementing larger case-control studies with SES-matched, population-based controls.

aDivision of Public Health Biology and Epidemiology, School of Public Health, University of California, Berkeley, CA ${ }^{b}$ Department of Pediatric Hematology/Oncology, Kaiser Permanente, Sacramento, CA Address correspondence to: Marilyn L. Kwan, PhD, University of California, Berkeley, Ste. 500, 2150 Shattuck Ave., Berkeley, CA 94720; tel. 510-642-6406; fax 510-643-1735; e-mail <kwan@uclink.berkeley.edu>.

(c)2004 Association of Schools of Public Health 
Leukemia is the leading cause of cancer morbidity among children younger than 15 years of age in the United States. ${ }^{1}$ Data from the Surveillance, Epidemiology, and End Results (SEER) registry suggest that acute lymphoblastic leukemia (ALL) accounted for $78 \%$ of all childhood leukemia cases diagnosed in the U.S. from 1975 through 1995, while acute myeloid leukemia (AML) accounted for 16\%. ${ }^{1}$ Incidence among children younger than 15 years of age showed a modest increase from 1975 through the late 1980 s but decreased slightly from 1989 to $1999 .^{2}$

Currently, the causes of childhood ALL are not well known, although sex, age, race, in utero ionizing radiation, and specific genetic syndromes have been consistently shown to be associated with risk for ALL. ${ }^{1}$ As for childhood AML, chemotherapeutic agents and in utero and postnatal ionizing radiation are recognized risk factors, and an association has been elucidated between race and risk of childhood AML. ${ }^{1}$ Because ALL encompasses a heterogeneous group of molecular subtypes, independent examination of etiologic and epidemiologic factors is required for each subtype., ${ }^{3,4}$ Furthermore, the clinical presentation of these molecular subtypes is also quite varied. ${ }^{3}$

A possible infectious etiology for the major subtype of ALL has been suggested by Greaves, ${ }^{3}$ who hypothesized that c-ALL (common B-cell precursor ALL) arises as a consequence of a rare, abnormal response to a nonspecific common infection. Two separate genetic events may lead to the presentation of c-ALL: an initial spontaneous event during the expansion of B-cell precursors pre- or perinatally and a subsequent event in the same mutant clone following antigenic challenge early in life (e.g., exposure to a common, nonspecific infection and the resulting stimulation of the young child's underdeveloped immune system). Support for this hypothesis is seen in genetic backtracking of the preleukemic clone TEL-AML1, a genetic determinant of c-ALL, using newborn blood spots. ${ }^{4}$

Speculation has focused recently on the role of breastfeeding in protecting children from disease. ${ }^{3,5,6}$ Human milk has long been recognized as providing numerous antimicrobial, anti-inflammatory, and immunomodulating agents. Many studies have shown conclusively that breastfeeding protects against acute gastrointestinal infections through transmission of maternal antibodies, macrophages, and lymphocytes. ${ }^{7,8}$ In contrast, the evidence for the protective effect of breastfeeding with regard to acute respiratory infection is still under debate. ${ }^{8,9}$ Therefore, since breast milk contains many beneficial biological factors, the Greaves hypothesis implies that breastfeeding mediates the occurrence of childhood ALL as a result of a rare, abnormal response to a common infection. ${ }^{1}$ To date, no similar mechanism has been suggested for the association of breastfeeding and childhood AML.

The scientific evidence has been mixed regarding the association of breastfeeding with childhood leukemia. A number of studies have shown no association between breastfeeding and leukemia risk. ${ }^{10-23}$ Five recent case-control studies, however, have suggested that breastfeeding protects children from developing childhood ALL. ${ }^{624-27}$ The largest of these studies ${ }^{24}$ included more than 1,700 ALL cases, while the smallest study ${ }^{27}$ included only 69 cases. In addition to varying samples sizes, these five studies differed on methods of case ascertainment and selection of controls, and in measurement of breastfeeding duration. More important, two out of the five studies ${ }^{25,27}$ did not adjust for any marker of socioeconomic status (SES). This lack of adjustment in the statistical analysis is unusual since measures of SES, particularly maternal education, have been shown to be highly associated with breastfeeding. ${ }^{28}$ As for the association of SES with risk of childhood leukemia, results have varied. Early ecologic and descriptive studies in the United States suggested that higher SES was a possible risk factor for childhood leukemia, but in contrast, case-control studies have reported lower SES in childhood leukemia patients compared to control subjects. ${ }^{29}$

The above issues prompt the need for a thorough review of the current literature on breastfeeding and childhood leukemia. For this study, we used a meta-analytic approach to evaluate the association of maternal breastfeeding with the risk of childhood leukemia. To our knowledge, this is the first report to systematically review epidemiologic data on this topic and to present risk estimates for childhood ALL and AML associated with breastfeeding. Our goals were (1) to quantify any association between duration of breastfeeding and risk of childhood ALL or AML, (2) to assess the influence of SES on any such associations, and (3) to discuss the implications of these findings for the evaluation of whether breastfeeding reduces the risk of childhood leukemia.

\section{METHODS}

We searched MEDLINE and CancerLit for original research and review articles on childhood leukemia and breastfeeding. In addition, we reviewed the bibliographies in these publications as well as master's theses and $\mathrm{PhD}$ dissertations filed electronically that addressed breastfeeding and childhood leukemia. Keyword combinations for the online searches consisted of "breastfeeding and childhood cancer," "breastfeeding and childhood leukemia," "infant feeding and childhood leukemia," "infant feeding and childhood cancer," "infant feeding and cancer," and the names of three prominent investigators in the field of maternal and child health whose names were encountered in the initial literature search.

Thirty articles were initially identified. Studies that presented data on any type of leukemia in children 15 years or younger in terms of an odds ratio (OR) and confidence interval (CI) and analyzed duration of breastfeeding in months were selected for the analysis. Seventeen studies were excluded, leaving 14 for the analysis. ${ }^{6,10-13,15,17,19-21,24-26,30}$ Three of these studies were not peer-reviewed. ${ }^{10,11,21}$ Of the 17 excluded articles, two reported no ORs, ${ }^{31,32}$ one reported no CIs or $p$-values, ${ }^{33}$ two reported ORs only for all cancers combined,,$^{34,35}$ three were duplicate reports, ${ }^{36-38}$ two were topical reviews on breastfeeding, ${ }^{9,39}$ one was a literature review, ${ }^{5}$ and four did not analyze breastfeeding duration. ${ }^{14,16,18,27} \mathrm{Fi}-$ nally, two cohort studies did not satisfy the selection criteria: one did not report any quantitative results ${ }^{22}$ while the other did not analyze breastfeeding duration. ${ }^{23}$

Table 1 outlines important characteristics of the studies included in the meta-analysis. First, eight of the 14 studies excluded cases of leukemia in infants (usually children younger than 1 year of age) to avoid possible biases associ- 
Table 1. Characteristics of 14 case-control studies included in meta-analysis examining the association between breastfeeding and risk of childhood leukemia

\begin{tabular}{|c|c|c|c|c|c|c|}
\hline Study & $\begin{array}{l}\text { Source of } \\
\text { cases }\end{array}$ & $\begin{array}{l}\text { Source of } \\
\text { controls }\end{array}$ & $\begin{array}{l}\text { Data collection } \\
\text { procedure }\end{array}$ & $\begin{array}{c}\text { OR } \\
(95 \% \mathrm{Cl})\end{array}$ & $\begin{array}{c}\text { Matching and } \\
\text { adjustment variables }\end{array}$ & Comments \\
\hline $\begin{array}{l}\text { van Duijn et al. } \\
1988 \text { (Netherlands) }{ }^{10}\end{array}$ & $\begin{array}{l}\text { National registry of } \\
\text { the Dutch Childhood } \\
\text { Leukemia Study } \\
\text { Group; } n=492 \mathrm{ALL} \\
\text { cases ( } 88 \% \\
\text { participation rate). }\end{array}$ & $\begin{array}{l}\text { Randomly } \\
\text { selected from } \\
\text { local municipal } \\
\text { registries; } n=480 \\
\text { ( } 67 \% \text { participation } \\
\text { rate). }\end{array}$ & $\begin{array}{l}\text { Questionnaire mailed } \\
\text { to parents. }\end{array}$ & $\begin{array}{l}\text { ALL: } \\
>6 \text { months vs. none: } \\
0.83(0.48,1.43) \\
\leq 6 \text { months vs. none: } \\
1.15(0.80,1.67)\end{array}$ & $\begin{array}{l}\text { Matching: age }( \pm 3 \\
\text { years), sex. } \\
\text { Adjustment: birth } \\
\text { order, social class, } \\
\text { maternal education, } \\
\text { maternal age, maternal } \\
\text { smoking during } \\
\text { pregnancy, maternal } \\
\text { alcohol use during } \\
\text { pregnancy. }\end{array}$ & $\begin{array}{l}\text { 1. Broad range of } \\
\text { matching on age } \\
\text { might be prone to } \\
\text { residual confounding. } \\
\text { 2. Cases in infants } \\
(<1.5 \text { years) excluded. } \\
\text { 3. Percentage of data } \\
\text { collected from mother } \\
\text { or father is unknown. }\end{array}$ \\
\hline $\begin{array}{l}\text { Magnani et al. } 1988 \\
\text { (Turin, Italy) }\end{array}$ & $\begin{array}{l}\text { Main pediatric } \\
\text { hospital of Turin; } 142 \\
\text { ALL and } 22 \mathrm{AML} \\
\text { cases (no } \\
\text { participation data } \\
\text { given). }\end{array}$ & $\begin{array}{l}\text { Random sample } \\
\text { of } 307 \text { children in } \\
\text { same hospital } \\
\text { with various } \\
\text { medical and } \\
\text { surgical } \\
\text { conditions (no } \\
\text { participation data } \\
\text { given). }\end{array}$ & $\begin{array}{l}\text { Interview with one } \\
\text { parent. }\end{array}$ & $\begin{array}{l}\text { ALL: } \\
>6 \text { months vs. none: } \\
1.06(0.54,2.08) ; \\
\leq 6 \text { months vs. none: } \\
1.10(0.74,1.64) \\
\text { ANLL: } \\
>6 \text { months vs. none: } \\
0.5 \\
\leq 6 \text { months vs. none: } \\
1.2 \text {. }\end{array}$ & $\begin{array}{l}\text { Matching: none. } \\
\text { Adjustment: SES, } \\
\text { maternal age. }\end{array}$ & $\begin{array}{l}\text { 1. Both incident and } \\
\text { prevalent cases used } \\
\text { in analysis. } \\
\text { 2. Cases came from } \\
\text { only one hospital. } \\
\text { 3. Age range of case } \\
\text { patients unknown. } \\
\text { 4. Small sample size, } \\
\text { especially for AML. }\end{array}$ \\
\hline $\begin{array}{l}\text { Davis et al. } 1988 \\
\text { (Denver, Colorado) }^{12}\end{array}$ & $\begin{array}{l}\text { Colorado Central } \\
\text { Cancer Registry and } \\
\text { review of hospital } \\
\text { records; } 52 \text { ALL and } \\
11 \text { "other" leukemia } \\
\text { cases ( } 71 \% \\
\text { participation rate). }\end{array}$ & $\begin{array}{l}\text { RDD; } n=63 \\
\text { (participation rate } \\
\text { not calculable). }\end{array}$ & $\begin{array}{l}\text { In-person interview } \\
\text { with mother. }\end{array}$ & $\begin{array}{l}\text { ALL: } \\
>6 \text { months vs. none: } \\
0.68(0.32,1.47) ; \\
\leq 6 \text { months vs. none: } \\
0.51(0.23,1.16) \text {. } \\
\text { Other leukemias: } \\
>6 \text { months vs. none: } \\
1.92(0.45,8.33) ; \\
\leq 6 \text { months vs. none: } \\
1.12(0.25,5.0) \text {. }\end{array}$ & $\begin{array}{l}\text { Matching: age }( \pm 3 \\
\text { years), sex, telephone } \\
\text { exchange. } \\
\text { Adjustment: none. }\end{array}$ & $\begin{array}{l}\text { 1. Small sample size. } \\
\text { 2. RDD potentially } \\
\text { produces selection } \\
\text { bias. } \\
\text { 3. No adjustment for } \\
\text { SES. } \\
\text { 4. Cases in infants } \\
\text { ( }<1.5 \text { years) excluded. } \\
\text { 5. Broad range of } \\
\text { matching on age } \\
\text { might be prone to } \\
\text { residual confounding. }\end{array}$ \\
\hline
\end{tabular}


Table 1 (continued). Characteristics of 14 case-control studies included in meta-analysis examining the association between breastfeeding and risk of childhood leukemia

\begin{tabular}{|c|c|c|c|c|c|c|}
\hline Study & $\begin{array}{l}\text { Source of } \\
\text { cases }\end{array}$ & $\begin{array}{l}\text { Source of } \\
\text { controls }\end{array}$ & $\begin{array}{l}\text { Data collection } \\
\text { procedure }\end{array}$ & $\begin{array}{c}\text { OR } \\
(95 \% \mathrm{Cl})\end{array}$ & $\begin{array}{c}\text { Matching and } \\
\text { adjustment variables }\end{array}$ & Comments \\
\hline 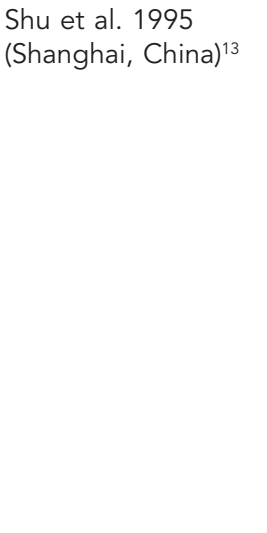 & $\begin{array}{l}\text { Shanghai Cancer } \\
\text { Registry; } 108 \mathrm{ALL} \\
\text { and } 51 \mathrm{AML} \text { cases } \\
\text { ( } 84 \% \text { participation } \\
\text { rate). }\end{array}$ & $\begin{array}{l}\text { Randomly } \\
\text { selected from } \\
\text { general } \\
\text { population of } \\
\text { urban Shanghai } \\
\text { residents, using } \\
\text { household group } \\
\text { as the sampling } \\
\text { unit; } n=159 \\
\text { (participation rate } \\
\text { not calculable). }\end{array}$ & $\begin{array}{l}\text { In-person interview } \\
\text { with parents. }\end{array}$ & $\begin{array}{l}\text { ALL: } \\
\text { ever vs. never: } \\
1.12(0.6,2.1) ; \\
>6 \text { months vs. none: } \\
1.12(0.6,2.2) ; \\
\leq 6 \text { months vs. none: } \\
1.10(0.5,2.5) \text {. } \\
\text { ANLL: } \\
\text { ever vs. never: } \\
1.34(0.5,3.3) ; \\
>6 \text { months vs. none: } \\
1.21(0.5,3.3) ; \\
\leq 6 \text { months vs. none: } \\
1.65(0.5,5.6) \text {. }\end{array}$ & $\begin{array}{l}\text { Matching: sex, year of } \\
\text { birth. } \\
\text { Adjustment: maternal } \\
\text { age at birth, } \\
\text { birthweight, maternal } \\
\text { working status, } \\
\text { maternal occupational } \\
\text { exposure to chemicals } \\
\text { during child's infancy. }\end{array}$ & $\begin{array}{l}\text { 1. Sampling unit was } \\
\text { an administrative entity } \\
\text { in the city of Shanghai. } \\
\text { 2. Small sample size. } \\
\text { 3. Cases in infants ( }<1 \\
\text { year) excluded. } \\
\text { 4. Defined the } \\
\text { exposure of interest } \\
\text { (breastfed defined as } \\
\text { having been fed any } \\
\text { amount of the natural } \\
\text { mother's breast milk } \\
\text { during infancy for any } \\
\text { duration). }\end{array}$ \\
\hline 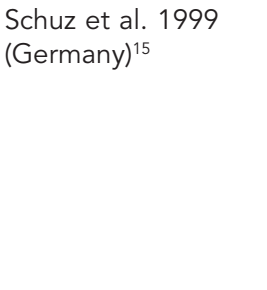 & $\begin{array}{l}\text { Nationwide German } \\
\text { Children's Cancer } \\
\text { Registry; } 682 \mathrm{ALL} \\
\text { and } 319 \text { "other" } \\
\text { leukemia cases (82\% } \\
\text { participation rate). }\end{array}$ & $\begin{array}{l}\text { Randomly } \\
\text { selected from } \\
\text { complete files of } \\
\text { local offices for } \\
\text { resident } \\
\text { registration; } \\
n=1,001 \text { ( } 69 \% \\
\text { participation rate). }\end{array}$ & $\begin{array}{l}\text { Mailed questionnaire } \\
\text { and telephone } \\
\text { interview with mother. }\end{array}$ & $\begin{array}{l}\text { ALL: } \\
>6 \text { months vs. } \\
\leq 1 \text { month: } \\
0.77(0.59,1.0) \\
\leq 6 \text { months vs. } \\
\leq 1 \text { month: } \\
0.83 \text { (0.63, 1.11). }\end{array}$ & $\begin{array}{l}\text { Matching: date of } \\
\text { birth, sex. } \\
\text { Adjustment: SES, } \\
\text { degree of } \\
\text { urbanization. }\end{array}$ & $\begin{array}{l}\text { 1. Large sample size. } \\
\text { 2. Baseline was } \leq 1 \\
\text { month of } \\
\text { breastfeeding, which } \\
\text { could have deflated } \\
\text { OR. }\end{array}$ \\
\hline $\begin{array}{l}\text { Children's Cancer } \\
\text { Group (CCG) study } \\
1999 \text { (U.S., Canada, } \\
\text { Australia) }\end{array}$ & $\begin{array}{l}\text { Registration files of } \\
\text { CCG; } 1,744 \text { ALL and } \\
456 \text { AML cases ( } 92 \% \\
\text { participation rate for } \\
\text { ALL and } 83 \% \\
\text { participation rate for } \\
\text { AML). }\end{array}$ & $\begin{array}{l}\mathrm{RDD} ; n=2418 \\
\text { (participation rate } \\
\text { not calculable). }\end{array}$ & $\begin{array}{l}\text { Telephone interview } \\
\text { with mother. }\end{array}$ & $\begin{array}{l}\text { ALL: } \\
\text { ever vs. never: } \\
0.80(0.69,0.93) ; \\
>6 \text { months vs. none: } \\
0.72(0.60,0.87) ; \\
\leq 6 \text { months vs. none: } \\
0.86(0.73,1.01) \text {. } \\
\text { AML: } \\
\text { ever vs. never: } \\
0.77(0.57,1.03) ; \\
>6 \text { months vs. none: } \\
0.57(0.39,0.84) ; \\
\leq 6 \text { months vs. none: } \\
0.95(0.68,1.33) \text {. }\end{array}$ & $\begin{array}{l}\text { Matching: age at } \\
\text { diagnosis within } 25 \% \\
\text { of the case age, } \\
\text { geographic location } \\
\text { (telephone area code } \\
\text { and exchange), race } \\
\text { (white vs. non-white). } \\
\text { Adjustment: maternal } \\
\text { race, maternal } \\
\text { education, family } \\
\text { annual income. }\end{array}$ & $\begin{array}{l}\text { 1. Large sample size. } \\
\text { 2. RDD has potential } \\
\text { to produce selection } \\
\text { bias. } \\
\text { 3. If child was } \\
\text { breastfed for }<1 \\
\text { month, he/she was } \\
\text { grouped into the not } \\
\text { breastfed group, which } \\
\text { could have deflated } \\
\text { OR. } \\
\text { 4. Also analyzed } \\
\text { breastfeeding in } \\
3-\text { month categories. } \\
5 . \text { Cases in infants } \\
\text { (<1 year) excluded } \\
\text { from analysis. }\end{array}$ \\
\hline
\end{tabular}


Table 1 (continued). Characteristics of 14 case-control studies included in meta-analysis examining the association

between breastfeeding and risk of childhood leukemia

\begin{tabular}{|c|c|c|c|c|c|c|}
\hline Study & $\begin{array}{c}\text { Source of } \\
\text { cases }\end{array}$ & $\begin{array}{l}\text { Source of } \\
\text { controls }\end{array}$ & $\begin{array}{l}\text { Data collection } \\
\text { procedure }\end{array}$ & $\begin{array}{c}\text { OR } \\
(95 \% \mathrm{Cl})\end{array}$ & $\begin{array}{c}\text { Matching and } \\
\text { adjustment variables }\end{array}$ & Comments \\
\hline $\begin{array}{l}\text { Dockerty et al. } 1999 \\
\text { (New Zealand) }{ }^{17}\end{array}$ & $\begin{array}{l}\text { National databases } \\
\text { including the New } \\
\text { Zealand Cancer } \\
\text { Registry; } 97 \text { ALL and } \\
24 \text { "other" leukemia } \\
\text { cases ( } 92 \% \\
\text { participation rate). }\end{array}$ & $\begin{array}{l}\text { National birth } \\
\text { records; } n=303 \\
\text { (participation rate } \\
\text { not calculable). }\end{array}$ & $\begin{array}{l}\text { In-person interview } \\
\text { with mother. }\end{array}$ & $\begin{array}{l}\text { ALL: } \\
\text { ever vs. never: } \\
0.98(0.39,2.47) ; \\
\text { > } 6 \text { months vs. none: } \\
0.62(0.28,1.38) ; \\
\leq 6 \text { months vs. none: } \\
1.24(0.47,3.23) \text {. }\end{array}$ & $\begin{array}{l}\text { Matching: age, sex. } \\
\text { Adjustment: age, sex, } \\
\text { child's social class, } \\
\text { mother's education, } \\
\text { household crowding, } \\
\text { delay from reference } \\
\text { date to interview. }\end{array}$ & $\begin{array}{l}\text { 1. The analysis was } \\
\text { done unmatched using } \\
\text { logistic regression. } \\
\text { 2. Small sample size. }\end{array}$ \\
\hline $\begin{array}{l}\text { Smulevich et al. } 1999 \\
\text { (Moscow, Russia) }\end{array}$ & $\begin{array}{l}\text { Records of Moscow } \\
\text { Central Cancer } \\
\text { Dispensary and the } \\
\text { registers of pediatric } \\
\text { clinics; } 109 \text { ALL, } 25 \\
\text { AML, and } 65 \text { "other" } \\
\text { leukemia cases } \\
\text { (participation rate not } \\
\text { calculable). }\end{array}$ & $\begin{array}{l}\text { Randomly } \\
\text { selected from the } \\
\text { register of the } \\
\text { local pediatric } \\
\text { polyclinic in the } \\
\text { area where the } \\
\text { case was diag- } \\
\text { nosed; } n=398 \\
\text { (participation rate } \\
\text { not calculable). }\end{array}$ & $\begin{array}{l}\text { In-person interviews } \\
\text { with parents ( } 96 \% \\
\text { mothers). }\end{array}$ & $\begin{array}{l}\text { Leukemia: } \\
\text { ever vs. never: } \\
0.64 \text { (0.41, } 1.00) ; \\
>6 \text { months vs. } \\
<2 \text { months: } \\
0.76 \text { (0.47, } 1.22) ; \\
2-6 \text { months vs. } \\
<2 \text { months: } \\
0.52(0.32,0.86)\end{array}$ & $\begin{array}{l}\text { Matching: age, sex, } \\
\text { and residence. } \\
\text { Adjustment: frequency } \\
\text { of alcohol intake by } \\
\text { parents; parental } \\
\text { smoking (when } \\
\text { univariate analyses } \\
\text { were significant). }\end{array}$ & $\begin{array}{l}\text { 1. Small sample size. } \\
\text { 2. No adjustment for } \\
\text { SES. }\end{array}$ \\
\hline $\begin{array}{l}\text { Infante-Rivard et al. } \\
2000 \text { (Quebec, } \\
\text { Canada) }{ }^{26}\end{array}$ & $\begin{array}{l}\text { Tertiary care centers; } \\
491 \text { ALL cases ( } 96 \% \\
\text { participation rate). }\end{array}$ & $\begin{array}{l}\text { Family allowance } \\
\text { files; } n=493 \text { ( } 84 \% \\
\text { participation rate). }\end{array}$ & $\begin{array}{l}\text { Telephone interview } \\
\text { with mother. }\end{array}$ & $\begin{array}{l}\text { ALL: } \\
>3 \text { months vs. none: } \\
0.67(0.47,0.94) ; \\
\leq 3 \text { months vs. none: } \\
0.68(0.49,0.95) \text {. }\end{array}$ & $\begin{array}{l}\text { Matching: age, sex, } \\
\text { region of residence at } \\
\text { the time of diagnosis. } \\
\text { Adjustment: maternal } \\
\text { age, maternal } \\
\text { education. }\end{array}$ & $\begin{array}{l}\text { 1. Case patients } \leq 9 \\
\text { years old. } \\
\text { 2. Breastfeeding not } \\
\text { categorized in the } \\
\text { standard way, i.e., } \\
\leq 6 \text { months vs. } \\
>6 \text { months). } \\
3.84 \% \text { of the case } \\
\text { interviews and } 86 \% \text { of } \\
\text { the control interviews } \\
\text { occurred within } 10 \\
\text { years of the date of } \\
\text { diagnosis. }\end{array}$ \\
\hline
\end{tabular}


Table 1 (continued). Characteristics of 14 case-control studies included in meta-analysis examining the association

between breastfeeding and risk of childhood leukemia

\begin{tabular}{|c|c|c|c|c|c|c|}
\hline Study & $\begin{array}{c}\text { Source of } \\
\text { cases }\end{array}$ & $\begin{array}{l}\text { Source of } \\
\text { controls }\end{array}$ & $\begin{array}{l}\text { Data collection } \\
\text { procedure }\end{array}$ & $\begin{array}{c}\text { OR } \\
(95 \% \mathrm{Cl})\end{array}$ & $\begin{array}{c}\text { Matching and } \\
\text { adjustment variables }\end{array}$ & Comments \\
\hline $\begin{array}{l}\text { United Kingdom } \\
\text { Childhood Cancer } \\
\text { Study (UKCCS) } 2001 \\
\text { (England, Wales, } \\
\text { Scotland) }{ }^{19}\end{array}$ & $\begin{array}{l}\text { National cancer } \\
\text { registry; 1,401 ALL } \\
\text { and } 214 \mathrm{AML} \text { cases } \\
\text { (87\% participation } \\
\text { rate). }\end{array}$ & $\begin{array}{l}\text { Family Health } \\
\text { Services } \\
\text { Authorities and } \\
\text { Health Boards; } \\
n=3274 \text { ( } 72 \% \\
\text { participation rate). }\end{array}$ & $\begin{array}{l}\text { In-person interview } \\
\text { with mother. }\end{array}$ & $\begin{array}{l}\text { ALL: } \\
\text { ever vs. never: } \\
0.78(0.58,1.05) ; \\
<1 \text { months vs. none: } \\
0.82(0.53,1.26) ; \\
1-6 \text { months vs. none: } \\
0.85(0.60,1.20) ; \\
\geq 7 \text { months vs. none: } \\
0.65 \text { (0.43, 1.00). } \\
\text { AML: } \\
\text { ever vs. never: } \\
0.91 \text { (0.81, } 1.04) ; \\
<1 \text { months vs. none: } \\
0.98 \text { (0.82, } 1.17) ; \\
1-6 \text { months vs. none: } \\
0.90 \text { (0.77, } 1.04) ; \\
\geq 7 \text { months vs. none: } \\
0.89 \text { (0.75, } 1.05) \text {. }\end{array}$ & $\begin{array}{l}\text { Matching: month and } \\
\text { year of birth, sex, } \\
\text { region of residence at } \\
\text { diagnosis. } \\
\text { Adjustment: age at } \\
\text { diagnosis, sex, region } \\
\text { of residence, birth } \\
\text { order, deprivation } \\
\text { index. }\end{array}$ & $\begin{array}{l}\text { 1. Large sample size. } \\
\text { 2. Well-designed and } \\
\text { conducted case- } \\
\text { control study. } \\
\text { 3. The analysis was } \\
\text { done unmatched using } \\
\text { logistic regression. } \\
\text { 4. Cases in infants ( }<1 \\
\text { year) excluded from } \\
\text { analysis. }\end{array}$ \\
\hline $\begin{array}{l}\text { Hardell et al. } 2001 \\
\text { (Sweden) }{ }^{20}\end{array}$ & $\begin{array}{l}\text { Swedish Cancer } \\
\text { Register; } 204 \text { ALL } \\
\text { and } 26 \text { AML cases } \\
\text { ( } 87 \% \text { participation } \\
\text { rate). }\end{array}$ & $\begin{array}{l}\text { Swedish Birth } \\
\text { Register; } n=235 \\
\text { (89\% participation } \\
\text { rate). }\end{array}$ & $\begin{array}{l}\text { Medical records from } \\
\text { Child Healthcare } \\
\text { Centers. }\end{array}$ & $\begin{array}{l}\text { ALL: } \\
\geq 6 \text { months vs. } \\
<1 \text { month: } \\
0.9(0.5,1.8) ; \\
1-5 \text { months vs. } \\
<1 \text { month: } \\
1.0(0.5,2.0) \\
\text { AML: } \\
\geq 6 \text { months vs. } \\
<1 \text { month: } \\
0.3(0.0,3.2) \\
1-5 \text { months vs. } \\
<1 \text { month: } \\
0.2(0.0,2.0) \text {. }\end{array}$ & $\begin{array}{l}\text { Matching: age, sex. } \\
\text { Adjustment: none. }\end{array}$ & $\begin{array}{l}\text { 1. Small sample size } \\
\text { for AML. } \\
\text { 2. No adjustment for } \\
\text { SES. } \\
\text { 3. Data collected only } \\
\text { from medical records. }\end{array}$ \\
\hline
\end{tabular}


Table 1 (continued). Characteristics of 14 case-control studies included in meta-analysis examining the association between breastfeeding and risk of childhood leukemia

\begin{tabular}{|c|c|c|c|c|c|c|}
\hline Study & $\begin{array}{l}\text { Source of } \\
\text { cases }\end{array}$ & $\begin{array}{l}\text { Source of } \\
\text { controls }\end{array}$ & $\begin{array}{l}\text { Data collection } \\
\text { procedure }\end{array}$ & $\begin{array}{c}\text { OR } \\
(95 \% \mathrm{Cl})\end{array}$ & $\begin{array}{c}\text { Matching and } \\
\text { adjustment variables }\end{array}$ & Comments \\
\hline $\begin{array}{l}\text { Perrillat et al. } 2002 \\
\text { (France) }^{6}\end{array}$ & $\begin{array}{l}\text { Hospitals in Lille, } \\
\text { Lyon, Nancy, and } \\
\text { Paris; } 218 \mathrm{ALL} \text { and } \\
28 \mathrm{AML} \text { cases } \\
\text { (participation rate not } \\
\text { calculable). }\end{array}$ & $\begin{array}{l}\text { Children treated in } \\
\text { the same hospital } \\
\text { as the case } \\
\text { patients, mainly in } \\
\text { orthopedic and } \\
\text { emergency } \\
\text { departments; } \\
n=235 \\
\text { (participation rate } \\
\text { not calculable). }\end{array}$ & $\begin{array}{l}\text { In-person interview } \\
\text { with mother. }\end{array}$ & $\begin{array}{l}\text { ALL: } \\
>6 \text { months vs. none: } \\
0.5(0.2,1.1) ; \\
\leq 6 \text { months vs. none: } \\
1.1(0.7,1.7) \text {. } \\
\text { ANLL: } \\
\text { >6 months vs. none: } \\
0.6(0.1,2.9) ; \\
\leq 6 \text { months vs. none: } \\
1.3(0.5,3.6) \text {. }\end{array}$ & $\begin{array}{l}\text { Matching: frequency } \\
\text { matched on age, sex, } \\
\text { hospital, hospital } \\
\text { catchment area, ethnic } \\
\text { origin. } \\
\text { Adjustment: age, } \\
\text { gender, hospital, } \\
\text { ethnic origin, maternal } \\
\text { education level, } \\
\text { parental socio- } \\
\text { professional category, } \\
\text { birthweight, length of } \\
\text { pregnancy, number of } \\
\text { previous pregnancies. }\end{array}$ & $\begin{array}{l}\text { 1. Children }<2 \text { years } \\
\text { of age excluded. } \\
\text { 2. Use of hospital } \\
\text { controls has potential } \\
\text { to produce Berkson's } \\
\text { bias. } \\
\text { 3. Adjustment for } \\
\text { many covariables. } \\
\text { 4. Small sample size } \\
\text { for AML. }\end{array}$ \\
\hline $\begin{array}{l}\text { Kwan } 2002 \\
\text { (San Francisco/ } \\
\text { Oakland Bay Area, } \\
\text { California) }\end{array}$ & $\begin{array}{l}\text { Rapid case } \\
\text { ascertainment from } 7 \\
\text { pediatric hospitals; } \\
147 \mathrm{ALL} \text { cases and } \\
31 \mathrm{AML} \text { cases ( } 83 \% \\
\text { participation rate). }\end{array}$ & $\begin{array}{l}\text { California birth } \\
\text { certificates from } \\
\text { Office of Vital } \\
\text { Records; } n=203 \\
(66 \%) .\end{array}$ & $\begin{array}{l}\text { Self-administered } \\
\text { questionnaire and in- } \\
\text { home interview with } \\
\text { biological mother } \\
\text { (95\%) or biological } \\
\text { father (5\%). }\end{array}$ & $\begin{array}{l}\text { ALL: } \\
>6 \text { months vs. none: } \\
1.03(0.51,2.08) ; \\
\leq 6 \text { months vs. none: } \\
0.87(0.43,1.77) \text {. } \\
\text { AML: } \\
>6 \text { months vs. none: } \\
0.38(0.08,1.93) ; \\
\leq 6 \text { months vs. none: } \\
0.29(0.06,1.54) \text {. }\end{array}$ & $\begin{array}{l}\text { Matching: age, sex, } \\
\text { maternal Hispanic } \\
\text { ethnicity, maternal } \\
\text { race, county of } \\
\text { residence at birth. } \\
\text { Adjustment: annual } \\
\text { household income. }\end{array}$ & $\begin{array}{l}\text { 1. Population-based } \\
\text { control selection } \\
\text { method. } \\
\text { 2. Cases in infants ( }<1 \\
\text { year) excluded from } \\
\text { analysis. } \\
\text { 3. Small sample size } \\
\text { for AML analysis. } \\
\text { 4. Detailed exposure } \\
\text { assessment. }\end{array}$ \\
\hline
\end{tabular}


Table 1 (continued). Characteristics of 14 case-control studies included in meta-analysis examining the association

between breastfeeding and risk of childhood leukemia

\begin{tabular}{|c|c|c|c|c|c|c|}
\hline Study & $\begin{array}{l}\text { Source of } \\
\text { cases }\end{array}$ & $\begin{array}{l}\text { Source of } \\
\text { controls }\end{array}$ & $\begin{array}{l}\text { Data collection } \\
\text { procedure }\end{array}$ & $\begin{array}{c}\text { OR } \\
(95 \% \mathrm{Cl})\end{array}$ & $\begin{array}{c}\text { Matching and } \\
\text { adjustment variables }\end{array}$ & Comments \\
\hline $\begin{array}{l}\text { Lancashire et al. } 2003 \\
\text { (England, Wales, } \\
\text { Scotland) })^{30}\end{array}$ & $\begin{array}{l}\text { National cancer } \\
\text { registry; } 948 \mathrm{ALL} \\
\text { cases and } 394 \mathrm{AML} \\
\text { cases for analysis } \\
(56 \% \text { participation } \\
\text { rate). }\end{array}$ & $\begin{array}{l}\text { Birth register of } \\
\text { the local authority } \\
\text { area in which the } \\
\text { case child died; } \\
n=3,827 \\
\text { (participation rate } \\
\text { not calculable). }\end{array}$ & $\begin{array}{l}\text { In-person interview } \\
\text { with parents. }\end{array}$ & $\begin{array}{l}\text { ALL: } \\
\geq 7 \text { months vs. none: } \\
0.90(0.60,1.34) ; \\
1-6 \text { months vs. none: } \\
0.96(0.77,1.20) \text {. } \\
\text { Other leukemias: } \\
\geq 7 \text { months vs. none: } \\
1.16(0.64,2.11) ; \\
1-6 \text { months vs. none: } \\
0.90(0.62,1.31) \text {. }\end{array}$ & $\begin{array}{l}\text { Matching: sex, date of } \\
\text { birth. } \\
\text { Adjustment: social } \\
\text { class based on } \\
\text { paternal occupation, } \\
\text { age of mother at birth } \\
\text { of index child, sibship } \\
\text { position. }\end{array}$ & $\begin{array}{l}\text { 1. Cases in infants }(<1 \\
\text { year) excluded from } \\
\text { analysis. } \\
\text { 2. Population-based } \\
\text { control selection } \\
\text { method. } \\
\text { 3. Data obtained after } \\
\text { death of child could } \\
\text { be prone to recall bias. } \\
\text { 4. Large sample size. }\end{array}$ \\
\hline
\end{tabular}

$\mathrm{OR}=$ odds ratio

$\mathrm{Cl}=$ confidence interval

SES = socioeconomic status

$\mathrm{ALL}=$ acute lymphoblastic leukemia

$A M L=$ acute myeloblastic leukemia

ANLL = acute non-lymphoblastic leukemia

$\mathrm{RDD}=$ random digit dialing 
ated with premature cessation of breastfeeding in children with cancer and also because most leukemias occurring during infancy are known to have different etiologies from childhood leukemias. ${ }^{40}$ We divided the 14 studies into subgroups based on the leukemia classifications used. The ALL classification was generally straightforward, with only one study specifying "leukemia" instead of "ALL." ${ }^{25}$ Since the majority of childhood leukemia is ALL, ${ }^{1}$ we included this study in the ALL group. Four studies ${ }^{6,12,13,30}$ used the classifications "other leukemias" or acute non-lymphoblastic leukemia (ANLL) instead of AML. Nevertheless, we included these studies in the AML group since the majority of such cases are AML cases. ${ }^{1}$ Finally, for purposes of this meta-analysis, we classified breastfeeding for six months or less as shortterm breastfeeding, and breastfeeding for more than six months as long-term breastfeeding.

For each of the 14 articles reviewed, an OR and its $95 \%$ CI were extracted. When available, ORs adjusted for SES were selected since SES was the most widely used potential confounder in these studies. A fixed effects model based on the general principles of Greenland ${ }^{41}$ was utilized since this model maintains a direct relationship between sample size and relative weight among smaller studies, unlike a random effects model. ${ }^{42}$ In addition, a fixed effects model, in contrast to a random effects model, assumes no heterogeneity between studies. ${ }^{41}$

Each OR was assigned a weight $\left(\mathrm{W}_{\mathrm{i}}\right)$ equal to the inverse square of its standard error $(\mathrm{SE}): \mathrm{W}_{\mathrm{i}}=1 /(\mathrm{SE})^{2}$. SEs were calculated by dividing the natural $\log$ of the ratio of the upper and lower $95 \%$ confidence limits (CLs) by 3.92: $\mathrm{SE}=\ln \left(\mathrm{CL}_{\text {upper }} / \mathrm{CL}_{\text {lower }}\right) / 3.92$. For each study, the weight was multiplied by the natural $\log$ of the risk ratio $\left(b_{i}\right)$ to give a summary measure $\left(\mathrm{W}_{\mathrm{i}} \mathrm{b}_{\mathrm{i}}\right)$. A combined summary was calculated by adding the summary measures and dividing by the sum of the weights: $\mathrm{b}=\Sigma \mathrm{W}_{\mathrm{i}} \mathrm{b}_{\mathrm{i}} / \Sigma \mathrm{W}_{\mathrm{i}}$. A summary OR was produced by taking the exponential of the combined summary: $\mathrm{OR}_{\text {sum }}=\exp ^{\mathrm{b}}$.

Heterogeneity among study results was assessed using the Chi-square statistic: $\chi^{2}=\Sigma W_{i}\left(b-b_{i}\right)^{2}$. When evidence of heterogeneity was present, the $95 \%$ CI of the summary OR was readjusted using new weights based on a random effects model that incorporated between-study heterogeneity. ${ }^{43}$

Finally, publication bias, the tendency of journals to publish only those studies reporting significant associations, was evaluated visually by using the traditional funnel graph method to display the distribution of all included studies by their point estimates and SEs. ${ }^{44}$ Smaller studies will naturally be less precise (have larger SEs), and therefore, by chance, the risk estimates will vary around the true point estimate to a greater extent than in larger studies. As demonstrated by Light and Pillemer, a symmetric funnel shape is formed when study results and sample sizes are plotted if no publication bias is present. ${ }^{44}$ If bias is present, the shape of the graph is skewed, indicating a correlation between point estimates and their SEs. In addition, as recommended by Begg and Mazumdar, we performed rank correlation tests to test for a significant relationship between the sample sizes and effect sizes of the studies. ${ }^{45}$ If a significant correlation does exist between these two factors, then publication bias is considered to be present.

\section{RESULTS}

The 14 articles on breastfeeding and childhood leukemia contributed 6,835 ALL cases and 1,216 AML cases. No evidence of publication bias was apparent for studies reporting results on the association between long-term breastfeeding and risk of ALL since the data points for these studies were fairly randomly distributed around the combined OR estimate (Figure 1) and the $p$-value for the rank correlation tests was nonsignificant $(p=0.58)$. Similarly, we found no evidence of publication bias for studies exploring the association between long-term breastfeeding and risk of AML (not shown, $p=0.46$ ).

As shown in Tables 2 and 3, a significant, negative association was observed between short-term breastfeeding and ALL $(\mathrm{OR}=0.88 ; 95 \%$ CI $0.80,0.96)$, but the AML results $(\mathrm{OR}=0.90 ; 95 \%$ CI $0.80,1.02)$ were not significant. Tables 2 and 3 and Figures 2 and 3 show a significant, negative association between long-term breastfeeding and ALL based on 14 studies $(\mathrm{OR}=0.76 ; 95 \%$ CI $0.68,0.84)$ and a significant, negative association between long-term breastfeeding and AML based on eight studies ( $\mathrm{OR}=0.85 ; 95 \%$ CI $0.73,0.98)$. Heterogeneity statistics for leukemia types and duration of breastfeeding were not significant ( $p$-values ranged from 0.13 to 0.28 ) except for the ALL analysis addressing shortterm breastfeeding $(p=0.03)$. For this point estimate, the adjusted $95 \%$ CI $(0.80,0.97)$ was almost exactly the same as the unadjusted $95 \%$ CI $(0.80,0.96)$. Therefore, use of the fixed effects model, which assumes no heterogeneity among studies, was appropriate. A random effects model yielded similar ORs and heterogeneity statistics (not shown). Overall, the combined ORs for all 14 studies supported a protective role for breastfeeding with regard to the risk of childhood leukemia.

Separate analyses were also conducted that addressed the association of breastfeeding duration with risk of ALL or AML using data adjusted and not adjusted for SES (Tables 2 and 3). The ORs for short-term breastfeeding and risk of ALL based on adjusted and unadjusted data were both in the protective direction, analogous to the OR for the 14 studies combined. For long-term breastfeeding and ALL, the ORs based on adjusted and unadjusted data were similar to the OR for the combined studies, although breastfeeding was no longer significantly protective in the unadjusted data $\left(\mathrm{OR}_{\text {unadjusted }}=0.78 ; 95 \%\right.$ CI $\left.0.55,1.10\right)$. For the AML studies, the ORs for short-term breastfeeding based on adjusted and unadjusted data were both suggestive of a protective effect, similar to the OR for the combined studies. For long-term breastfeeding and risk of AML, the ORs for adjusted and unadjusted data were comparable to the OR for combined data, although breastfeeding was no longer significantly protective in the unadjusted data $\left(\mathrm{OR}_{\text {unadjusted }}=0.89,95 \% \mathrm{CI}\right.$ $0.29,2.71)$. The estimates for the unadjusted AML data should be interpreted with caution since only two studies were available for comparison. Table 4 shows the ORs for SES-adjusted data from the meta-analysis, as well as separately for the United Kingdom Childhood Cancer Study (UKCCS) and the Children's Cancer Group (CCG) study. ${ }^{19,24}$ Overall, SES as a potential confounder appeared to play no substantial role in the findings of either the short-term or long-term breastfeeding studies. 
Figure 1. Funnel graph to assess publication bias in studies of association between breastfeeding $>6$ months and risk of childhood ALL ( $n=14$ studies)

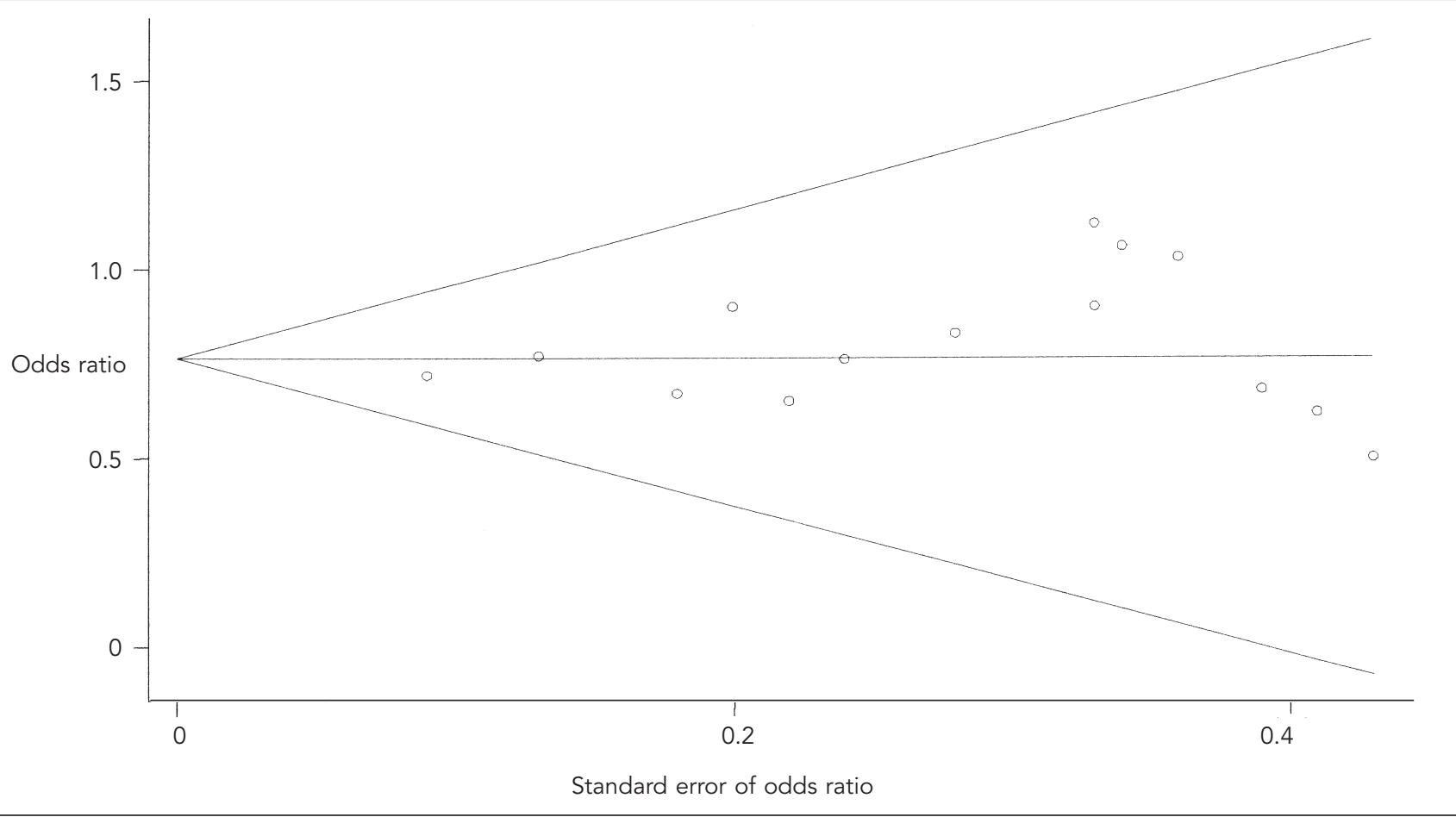

$\mathrm{ALL}=$ acute lymphoblastic leukemia

Tables 2 and 3 also display the weights applied to each study result in the meta-analysis. An important point to note is that the weights are directly related to the SE for each study (see Methods), but the SE does not always correlate with sample size-the SE depends on both the number of individuals in the study and the distribution of these individuals across exposure and disease categories. For ALL, no study contributed more than $34 \%$ of the total weight for long-term breastfeeding or more than $32 \%$ of the total weight for short-term breastfeeding. For AML, the $\mathrm{UKCCS}^{19}$ contributed $74 \%$ of the total weight for long-term breastfeeding and $70 \%$ of the total weight for short-term breastfeeding. Removal of this study from the AML analysis resulted in an OR of 0.73 (95\% CI $0.55,0.97)$ for long-term breastfeeding and an OR of $0.91(95 \%$ CI $0.73,1.15)$ for short-term breastfeeding. Thus, exclusion of this study produced a moderate decrease in the combined OR and a wider CI for long-term breastfeeding, and essentially no change in the combined OR and a wider CI for short-term breastfeeding.

\section{DISCUSSION}

This meta-analysis supports the hypothesis that short- and long-term breastfeeding play a protective role with regard to the risk for ALL. In addition-and not anticipated on biological grounds-the results show that long-term breastfeeding is also protective against AML. However, while the classification of ALL was fairly uniform across studies, the AML category was more variable. The variability in AML classification could lead to misclassification of the outcome and subsequent spurious associations. Furthermore, inclusion of SES as a potential confounder had minimal influence on the risk estimates for both short-term and long-term breastfeeding for ALL and AML. Before any general conclusions can be drawn, several issues must be considered: the results of the two largest case-control studies in the metaanalysis, ${ }^{19,24}$ the results of the two cohort studies, ${ }^{22,23}$ current knowledge regarding the etiology of ALL and AML, the relationship between SES and breastfeeding, and the effect of differential participation rates for case and control samples that differed in SES.

The UKCCS ${ }^{19}$ and CCG study ${ }^{24}$ were the two largest and/ or most influential studies in the meta-analysis. The UKCCS included 1,401 (87\%) ALL and $214(13 \%)$ AML case patients recruited from health programs that enrolled $98 \%$ of the total UK population; control subjects were selected from population-based health rosters. In contrast, the CCG study included 1,744 (79\%) ALL and 456 (21\%) AML case patients enrolled from specific CCG centers, and control subjects were selected via random-digit-dialing (RDD). The use of RDD in control recruitment introduces a concern regarding unknown and possibly stronger SES differentials between cases and controls, a major issue when conducting a case-control study. Both studies found that long-term breast- 
Table 2. Selected characteristics of 14 case-control studies included in the meta-analysis examining the association between breastfeeding and risk of childhood ALL, by duration of breastfeeding

\begin{tabular}{|c|c|c|c|c|c|}
\hline Study & SES-adjusted & Number of cases ( $n$ ) & $O R$ & $95 \% \mathrm{Cl}$ & $W_{i}(\%)$ \\
\hline \multicolumn{6}{|l|}{ Breastfeeding $\leq 6$ months } \\
\hline van Duijn et al. $1988^{10}$ & Yes & 492 & 1.15 & $0.80,1.67$ & $28.4(6)$ \\
\hline Magnani et al. $1988^{11}$ & Yes & 142 & 1.10 & $0.74,1.64$ & $24.3(5)$ \\
\hline Davis et al. $1988^{12}$ & No & 52 & 0.51 & $0.23,1.16$ & $5.9(1)$ \\
\hline Shu et al. $1995^{13}$ & Yes & 108 & 1.10 & $0.50,2.50$ & $5.9(1)$ \\
\hline Schuz et al. $1999^{15}$ & Yes & 682 & 0.83 & $0.63,1.11$ & $47.9(10)$ \\
\hline CCG study $1999^{24}$ & Yes & 1,744 & 0.86 & $0.73,1.01$ & $145.8(32)$ \\
\hline Dockerty et al. 199917 & Yes & 97 & 1.24 & $0.47,3.23$ & $4.1(1)$ \\
\hline Smulevich et al. $1999^{25}$ & No & 109 & 0.52 & $0.32,0.86$ & $15.7(3)$ \\
\hline Infante-Rivard et al. $2000^{26}$ & Yes & 491 & 0.68 & $0.49,0.95$ & $35.1(8)$ \\
\hline UKCCS $2001^{19}$ & Yes & 1401 & 0.85 & $0.60,1.20$ & $32.0(7)$ \\
\hline Hardell et al. $2001^{20}$ & No & 204 & 1.00 & $0.50,2.00$ & $8.0(2)$ \\
\hline Perrillat et al. $2002^{6}$ & Yes & 218 & 1.10 & $0.70,1.70$ & $19.5(4)$ \\
\hline Kwan $2002^{21}$ & Yes & 147 & 0.87 & $0.43,1.77$ & $7.4(2)$ \\
\hline Lancashire et al. $2003^{30}$ & Yes & 948 & 0.96 & $0.77,1.20$ & $78.1(17)$ \\
\hline Combined OR: SES-adjusted & - & 6,470 & 0.90 & $0.82,0.99$ & - \\
\hline Combined OR: not SES-adjusted & - & 365 & 0.62 & $0.43,0.89$ & - \\
\hline Combined OR: all studies & - & 6,835 & 0.88 & $0.80,0.97$ & $458.27(100)$ \\
\hline \multicolumn{6}{|l|}{ Breastfeeding $>6$ months } \\
\hline van Duijn et al. $1988^{10}$ & Yes & 492 & 0.83 & $0.48,1.43$ & $12.9(4)$ \\
\hline Magnani et al. $1988^{11}$ & Yes & 142 & 1.06 & $0.54,2.08$ & $8.4(3)$ \\
\hline Davis et al. $1988^{12}$ & No & 52 & 0.68 & $0.32,1.47$ & $6.6(2)$ \\
\hline Shu et al. $1995^{13}$ & Yes & 108 & 1.12 & $0.60,2.20$ & $9.1(3)$ \\
\hline Schuz et al. $1999^{15}$ & Yes & 682 & 0.77 & $0.59,1.00$ & $55.2(17)$ \\
\hline CCG study $1999^{24}$ & Yes & 1,744 & 0.72 & $0.60,0.87$ & $111.3(34)$ \\
\hline Dockerty et al. 199917 & Yes & 97 & 0.62 & $0.28,1.38$ & $6.0(2)$ \\
\hline Smulevich et al. $1999^{25}$ & No & 109 & 0.76 & $0.47,1.22$ & $16.9(5)$ \\
\hline Infante-Rivard et al. $2000^{26}$ & Yes & 491 & 0.67 & $0.47,0.94$ & $32.0(10)$ \\
\hline UKCCS $2001^{19}$ & Yes & 1,401 & 0.65 & $0.43,1.00$ & $21.6(7)$ \\
\hline Hardell et al. $2001^{20}$ & No & 204 & 0.90 & $0.50,1.80$ & $9.4(3)$ \\
\hline Perrillat et al. $2002^{6}$ & Yes & 218 & 0.50 & $0.20,1.10$ & $5.3(2)$ \\
\hline Kwan $2002^{21}$ & Yes & 147 & 1.03 & $0.51,2.08$ & $7.3(2)$ \\
\hline Lancashire et al. $2003^{30}$ & Yes & 948 & 0.90 & $0.60,1.34$ & $23.8(7)$ \\
\hline Combined OR: SES-adjusted & - & 6,470 & 0.75 & $0.67,0.85$ & - \\
\hline Combined OR: not SES-adjusted & - & 365 & 0.78 & $0.55,1.10$ & - \\
\hline Combined OR: all studies & - & 6,835 & 0.76 & $0.68,0.84$ & $326.27(100)$ \\
\hline \multicolumn{6}{|l|}{$\overline{\mathrm{ALL}}=$ acute lymphoblastic leukemia } \\
\hline \multicolumn{6}{|l|}{$\mathrm{SES}=$ socioeconomic status } \\
\hline \multicolumn{6}{|l|}{$\mathrm{OR}=$ odds ratio } \\
\hline $\mathrm{Cl}=$ confidence interval & & & & & \\
\hline$W_{i}=$ weight assigned to each odds $r$ & d & & & & \\
\hline
\end{tabular}

feeding was protective for ALL, but the CI for the risk estimate from the UKCCS did not exclude unity (1.00), whereas the CI for the risk estimate reported by the CCG study clearly excluded unity (1.00), indicating statistical significance. The UKCCS, however, found no association between short- or long-term breastfeeding and risk of AML, while the CCG study found a significant protective effect of long-term breastfeeding for AML. The UKCCS investigators were cautious in drawing conclusions due to a concern about systematic bias resulting from documented differences in participation between cases and controls. The CCG study investigators were less tenuous in their conclusion that the results of their study demonstrated lower risk of ALL and AML for breastfed infants, particularly those breastfed for more than six months.

Two large cohort studies have been published, one evaluating the association between breastfeeding and risk of childhood cancer ${ }^{22}$ and the other evaluating the association between breastfeeding and risk of childhood ALL. ${ }^{23}$ The major advantage of cohort studies is that they are not subject to the same biases as case-control studies and can better assess the temporal relationship between exposure and disease. Murray et al. conducted a historical cohort study of 434,933 singleton live births in Northern Ireland from 1971 through $1986 .{ }^{23}$ 
Table 3. Selected characteristics of 8 case-control studies included in the meta-analysis examining association between breastfeeding and risk of childhood AML, by duration of breastfeeding

\begin{tabular}{|c|c|c|c|c|c|}
\hline Study & SES-adjusted & Number of cases ( $\mathrm{n}$ ) & $O R$ & $95 \% \mathrm{Cl}$ & $W_{i}(\%)$ \\
\hline \multicolumn{6}{|l|}{ Breastfeeding $\leq 6$ months } \\
\hline Davis et al. $1988^{12}$ & No & 11 & 1.12 & $0.25,5.00$ & $1.7(1)$ \\
\hline Shu et al. $1995^{13}$ & Yes & 51 & 1.65 & $0.50,5.60$ & $2.6(1)$ \\
\hline CCG study $1999^{24}$ & Yes & 456 & 0.95 & $0.68,1.33$ & $34.1(14)$ \\
\hline UKCCS $2001^{19}$ & Yes & 214 & 0.90 & $0.77,1.04$ & $170.1(70)$ \\
\hline Hardell et al. $2001^{20}$ & No & 26 & 0.20 & $0.10,2.00$ & $1.7(1)$ \\
\hline Perrillat et al. $2002^{6}$ & Yes & 28 & 1.30 & $0.50,3.60$ & $3.9(2)$ \\
\hline Kwan $2002^{21}$ & Yes & 36 & 0.29 & $0.06,1.54$ & $1.5(1)$ \\
\hline Lancashire et al. $2003^{30}$ & Yes & 394 & 0.90 & $0.62,1.31$ & $27.5(11)$ \\
\hline Combined OR: SES-adjusted & - & 1,179 & 0.91 & $0.80,1.04$ & - \\
\hline Combined OR: not SES-adjusted & - & 37 & 0.47 & $0.16,1.36$ & - \\
\hline Combined OR: all studies & - & 1,216 & 0.90 & $0.80,1.02$ & $243.14(100)$ \\
\hline \multicolumn{6}{|l|}{ Breastfeeding $>6$ months } \\
\hline Davis et al. $1988^{12}$ & No & 11 & 1.92 & $0.45,8.33$ & $1.8(1)$ \\
\hline Shu et al. $1995^{13}$ & Yes & 51 & 1.21 & $0.50,3.30$ & $4.3(2)$ \\
\hline CCG study $1999^{24}$ & Yes & 456 & 0.57 & $0.39,0.84$ & $26.1(14)$ \\
\hline UKCCS $2001^{19}$ & Yes & 214 & 0.89 & $0.75,1.05$ & $135.7(74)$ \\
\hline Hardell et al. $2001^{20}$ & No & 26 & 0.30 & $0.10,3.20$ & $1.3(1)$ \\
\hline Perrillat et al. $2002^{6}$ & Yes & 28 & 0.60 & $0.10,2.90$ & $1.4(1)$ \\
\hline Kwan $2002^{21}$ & Yes & 36 & 0.38 & $0.08,1.93$ & $1.5(1)$ \\
\hline Lancashire et al. $2003^{30}$ & Yes & 394 & 0.85 & $0.73,0.98$ & $10.8(6)$ \\
\hline Combined OR: SES-adjusted & - & 1,179 & 0.85 & $0.73,0.98$ & - \\
\hline Combined OR: not SES-adjusted & - & 37 & 0.89 & $0.29,2.71$ & - \\
\hline Combined OR: all studies & - & 1,216 & 0.85 & $0.73,0.98$ & $182.90(100)$ \\
\hline \multicolumn{6}{|l|}{$\mathrm{AML}=$ acute myeloblastic leukemia } \\
\hline \multicolumn{6}{|l|}{$\mathrm{SES}=$ socioeconomic status } \\
\hline \multicolumn{6}{|l|}{$\mathrm{OR}=$ odds ratio } \\
\hline $\mathrm{Cl}=$ confidence interval & & & & & \\
\hline
\end{tabular}

Cases of ALL were identified through the Northern Ireland Child Health System and Northern Ireland Cancer Registry's Register of Childhood Cancers. No statistically significant difference was found for ever vs. never breastfeeding $(\mathrm{OR}=$ 0.98; $95 \%$ CI $0.68,1.42$ ) for ALL. Golding et al. conducted a national cohort study of 16,193 infants delivered in one week of April 1970 in Great Britain, following them until 1980. ${ }^{22}$ Thirty-three cancer cases, nine of which were leukemia cases, were identified from death certificates, through the Cancer Registration system, or from follow-up interviews conducted at ages 5 and 10 years. No childhood cancer data are presented by the investigators, but breastfeeding was reported to not be protective for childhood cancer. Although the Golding et al. study is not as informative as the study by Murray et al., these cohort studies indicate the lack of a protective effect of breastfeeding on risk of childhood ALL or childhood cancer.

The observation of a protective effect of breastfeeding for both ALL and AML in our analyses raises questions about the classification of leukemia cases in the two subgroups as well as the underlying biological mechanism involved. First, four studies included in this meta-analysis specified ANLL or "other leukemias" rather than AML ex- plicitly. ${ }^{6,12,13,30}$ Therefore, this group of leukemias might not be as homogeneous as the ALL group, thus constraining inferences regarding AML and breastfeeding. Second, Greaves's hypothesis of an abnormal immunological response leading to presentation with childhood leukemia is specific for the natural history of c-ALL. ALL and AML have different cell origins: childhood ALL arises from lymphoid stem cells while childhood AML arises from myeloid stem cells. ${ }^{46}$ If breastfeeding modulates the immune response via B-cell precursors, as the Greaves hypothesis suggests, then the protective effect observed for AML is unexpected. Our results imply that a separate immunological mechanism is operating via myeloid precursors along with the mechanism suggested by Greaves.

It is recognized that higher SES mothers have higher rates of breastfeeding than lower SES mothers, and this pattern has been stable over the past two decades. ${ }^{47,48}$ Higher SES mothers tend to retrospectively recall longer periods of breastfeeding than lower SES mothers. ${ }^{49}$ Variations in breastfeeding practices may reflect differences in education, a measure of SES. If the mother is better educated about the benefits of breast milk, she may be more inclined to breastfeed her child. ${ }^{28}$ In addition, lower SES mothers may 
Figure 2. Odds ratios (ORs) and $95 \%$ confidence intervals (Cls) in studies of association between breastfeeding $>6$ months and risk of childhood ALL ( $n=14$ studies)

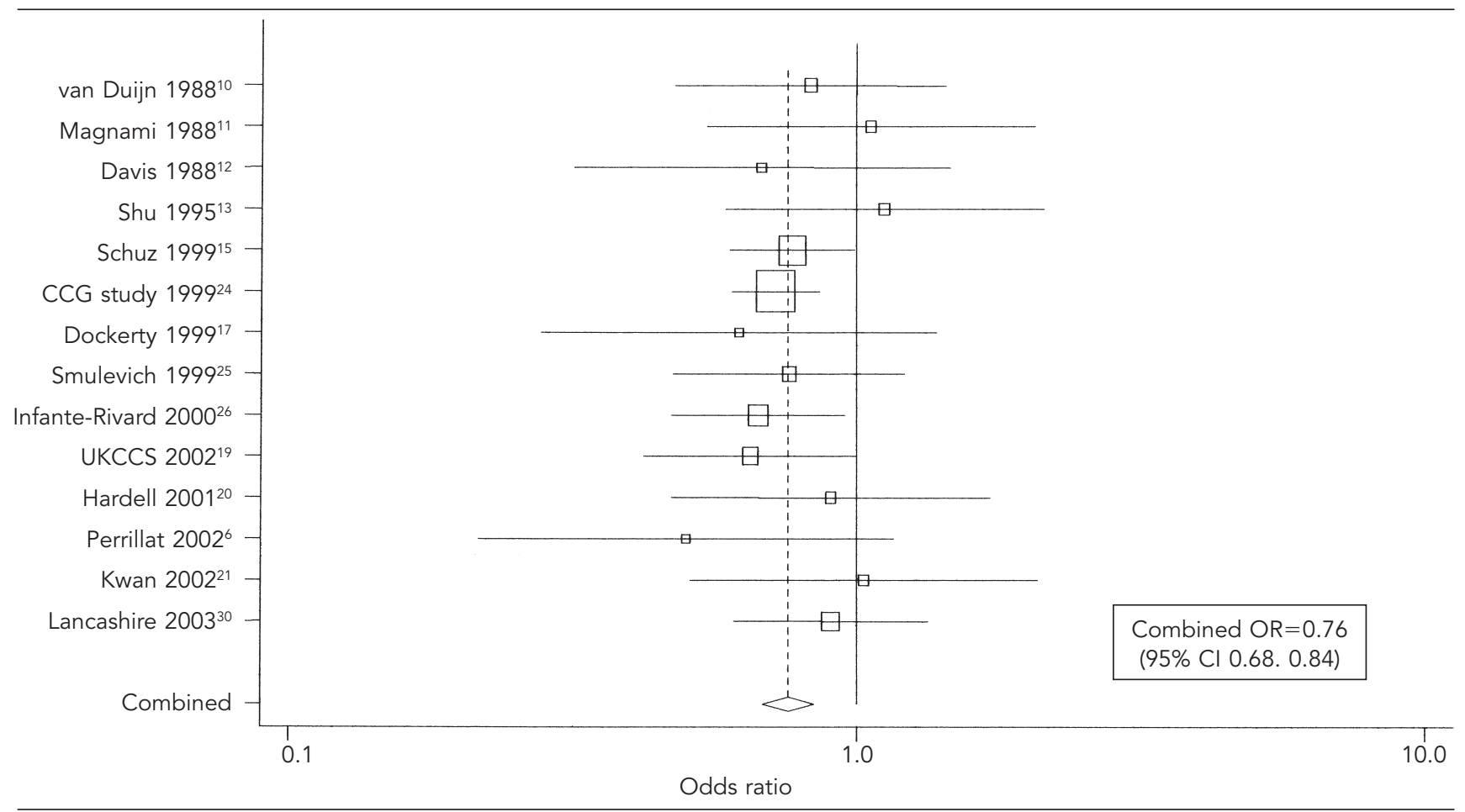

$\mathrm{ALL}=$ acute lymphoblastic leukemia

qualify for government food support programs that can help pay for infant formula, and the availability of food assistance for the family may deter the mother from breastfeeding her infant children. ${ }^{50}$

Finally, the roles of SES and participation bias can be assessed in this body of literature. In both the UKCCS ${ }^{19}$ and CCG study, ${ }^{24}$ the control subjects were of higher SES than the case patients. Even though SES was adjusted for in both of these studies (the UKCCS adjusted for a deprivation index, and the CCG study adjusted for maternal education and family annual income), this factor could have introduced some residual bias. The most important consideration is the representativeness of the controls relative to the base population from which the cases arise. This can best be assessed with the population-based design of studies such as the UKCCS.

The effect of differential participation rates between cases and controls on the overall results is important to consider. For example, when the UKCCS analysis was published, ${ }^{19}$ the authors cited a meta-analysis that demonstrated a protective role of breastfeeding with respect to risk of diabetes mellitus; however, the controls had higher participation rates than the cases. ${ }^{51}$ In the current meta-analysis, participation rates $^{52}$ could be calculated for only six of the 14 included studies. ${ }^{10,15,19-21,26}$ For five of these six studies, ${ }^{10,15,19,21,26}$ the participation rates for cases were higher than those for controls, and in most of these studies, the controls were of
Table 4. SES-adjusted risk of ALL and AML, by duration of breastfeeding: meta-analysis, UKCCS, ${ }^{19}$ and CCG study ${ }^{24}$

\begin{tabular}{|c|c|c|}
\hline \multirow{2}{*}{$\begin{array}{l}\text { Duration of } \\
\text { breastfeeding }\end{array}$} & \multicolumn{2}{|c|}{ OR $(95 \% \mathrm{Cl})$} \\
\hline & $A L L$ & $A M L$ \\
\hline Meta-analysis & $\underline{n=6,470(85 \%)}$ & $\underline{n=1,179(15 \%)}$ \\
\hline$\leq 6$ months & $0.90(0.82,0.99)$ & $0.91(0.80,1.04)$ \\
\hline$>6$ months & $0.75(0.67,0.85)$ & $0.85(0.73,0.98)$ \\
\hline UKCCS $2001^{19}$ & $\underline{n=1,401(87 \%)}$ & $n=214(13 \%)$ \\
\hline$\leq 6$ months & $0.85(0.60,1.20)$ & $0 . \overline{90}(0.77,1.04)$ \\
\hline$>6$ months & $0.65(0.43,1.00)$ & $0.89(0.75,1.05)$ \\
\hline CCG study $1999^{24}$ & $n=1,744(79 \%)$ & $n=456(21 \%)$ \\
\hline$\leq 6$ months & $0.86(0.73,1.01)$ & $0.95(0.68,1.33)$ \\
\hline$>6$ months & $0.72(0.60,0.87)$ & $0.57(0.39,0.84)$ \\
\hline \multicolumn{3}{|c|}{ SES $=$ socioeconomic status } \\
\hline \multicolumn{3}{|c|}{$\mathrm{ALL}=$ acute lymphoblastic leukemia } \\
\hline \multicolumn{3}{|c|}{$\mathrm{AML}=$ acute myeloblastic leukemia } \\
\hline \multicolumn{3}{|c|}{ UKCCS $=$ United Kingdom Childhood Cancer Study } \\
\hline \multicolumn{3}{|c|}{ CCG $=$ Children's Cancer Group } \\
\hline \multicolumn{3}{|l|}{$\mathrm{OR}=$ odds ratio } \\
\hline $\mathrm{Cl}=$ confidence inte & & \\
\hline
\end{tabular}


Figure 3. Odds ratios (ORs) and $95 \%$ confidence intervals (Cls) in studies of association between breastfeeding $>6$ months and risk of childhood AML ( $n=8$ studies)

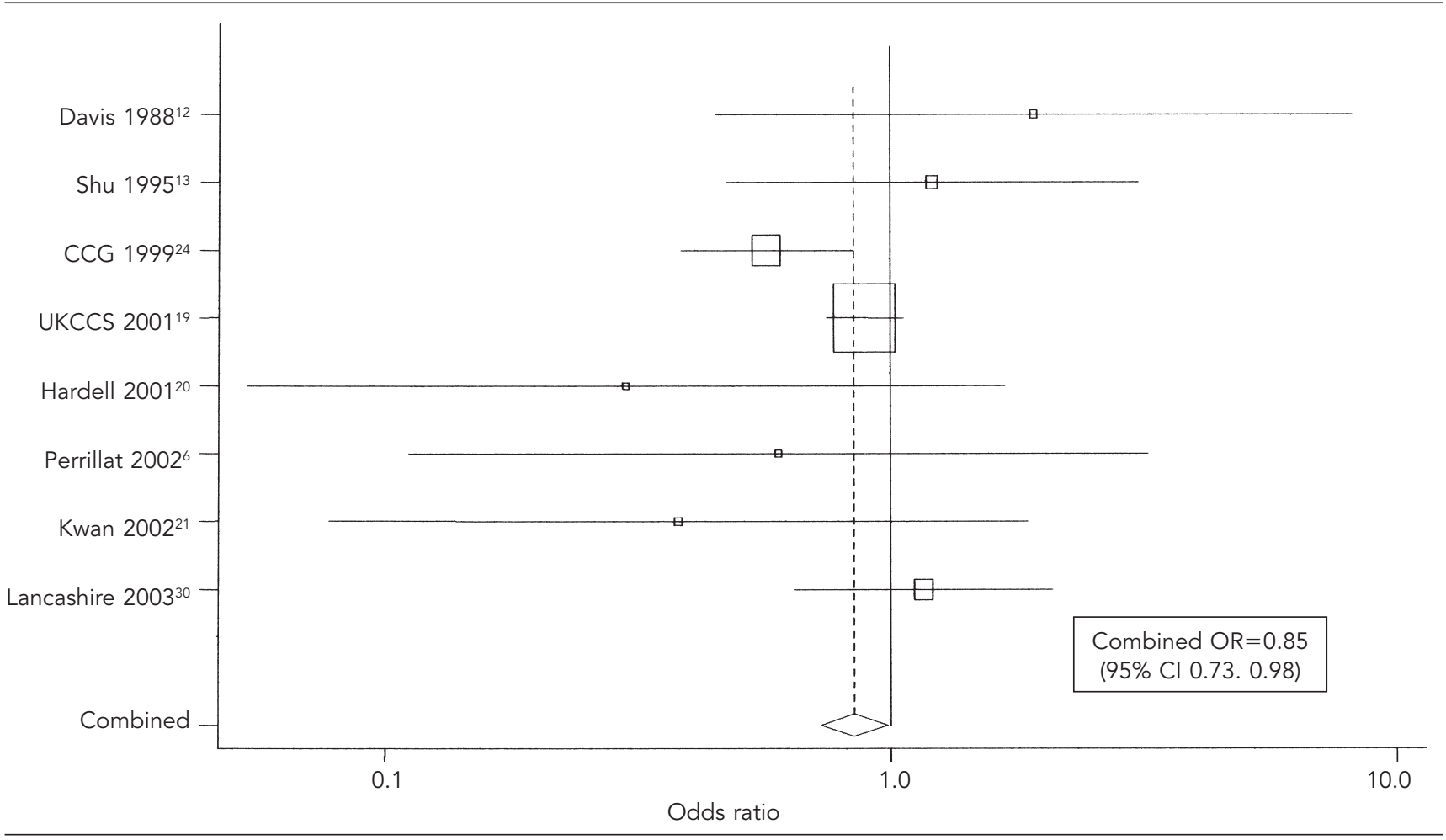

$\mathrm{AML}=$ acute myeloblastic leukemia

higher SES than the cases. Therefore, the association of breastfeeding with SES in combination with differential participation rates by SES could have biased the OR toward a protective effect.

This meta-analysis demonstrated a protective association between breastfeeding and risk of childhood ALL and possibly AML. Three alternative explanations must be considered. First, a systematic bias may be present in case-control studies of childhood leukemia arising from differential participation rates for case and control samples that differed in SES. Second, the effect on ALL risk may be spurious, as suggested by the results of the two cohort studies; ${ }^{22,23}$ unfortunately, these studies provided no information regarding AML. Third, the protective effect of breastfeeding may not be limited to ALL, as predicted by the Greaves hypothesis; however, support for a different underlying mechanism for AML would rely on an accurate and homogeneous category of AML cases. Further evaluation of the biological mechanisms while taking into consideration potential biases can be feasibly achieved with more large-scale case-control studies utilizing population-based, SES-matched controls.

In conclusion, the potential protective effect of breastfeeding on risk of childhood ALL may be more complicated than the current literature suggests. Nevertheless, the available evidence suggests that such a protective effect exists for both ALL and AML, with the caveats noted.
This study was supported by research grants from the National Institute of Environmental Health Sciences (PS42 ES04705 and R01 ES09137). The authors thank Craig Steinmaus, MD, MPH, for his guidance on the conduct of meta-analyses.

Author note: This meta-analysis does not include the study by Jourdan-Da Silva et al. (Jourdan-Da Silva, N., et al. Infectious diseases in the first year of life, perinatal characteristics and childhood acute leukaemia. Br J Cancer 2004;90:139-45) since their study was released after this manuscript's original submission in 2003. The authors re-calculated the combined ORs with the Jourdan-Da Silva paper, and these results were virtually the same as the results cited in the meta-analysis: combined OR for ALL and breastfeeding $\leq 6$ months $=0.89 ; 95 \%$ CI $0.82,0.97$; combined OR for ALL and breastfeeding $>6$ months $=0.76 ; 95 \%$ CI 0.69 , 0.85 ; combined OR for AML and breastfeeding $\leq 6$ months $=0.91$; 95\% CI 0.80, 1.03; combined OR for AML and breastfeeding $>6$ months $=0.85 ; 95 \%$ CI $0.73,0.98$.

\section{REFERENCES}

1. Smith M, Ries L, Gurney J, Ross J. Leukemia. In: Ries L, Smith M, Gurney J, Linet M, et al., editors. Cancer incidence and survival among children and adolescents: United States SEER program 1975-1995. Bethesda (MD): National Cancer Institute, SEER Program; 1999. p. 17-34.

2. Linet MS, Ries LA, Smith MA, Tarone RE, Devesa SS. Cancer surveillance series: recent trends in childhood cancer incidence and mortality in the United States. J Natl Cancer Inst 1999;91: 1051-8. 
3. Greaves M. Molecular genetics, natural history and the demise of childhood leukaemia. Eur J Cancer 1999;35:1941-53.

4. Wiemels JL, Cazzaniga G, Daniotti M, Eden OB, Addison GM, Masera G, et al. Prenatal origin of acute lymphoblastic leukaemia in children. Lancet 1999;354:1499-503.

5. Davis MK. Review of the evidence for an association between infant feeding and childhood cancer. Int J Cancer Suppl 1998;11:29-33.

6. Perrillat F, Clavel J, Jaussent I, Baruchel A, Leverger G, Nelken B, et al. Breast-feeding, fetal loss and childhood acute leukaemia. Eur J Pediatr 2002;161:235-7.

7. Goldman AS. The immune system of human milk: antimicrobial, antiinflammatory and immunomodulating properties. Pediatr Infect Dis J 1993;12:664-71.

8. Kramer MS, Chalmers B, Hodnett ED, Sevkovskaya Z, Dzikovich I, Shapiro S, et al. Promotion of Breastfeeding Intervention Trial (PROBIT): a randomized trial in the Republic of Belarus. JAMA 2001;285:413-20.

9. Villalpando S, Hamosh M. Early and late effects of breast-feeding: does breast-feeding really matter? Biol Neonate 1998;74:177-91.

10. van Duijn CM, van Steensel-Moll HA, van der Does-vd Berg A, van Wering ER, van Zanen GE, Valkenburg HA, Rammeloo JA. Infant feeding and childhood cancer [letter]. Lancet 1988;2:796-7.

11. Magnani C, Pastore G, Terracini B. Infant feeding and childhood cancer [letter]. Lancet 1988;2:1136.

12. Davis MK, Savitz DA, Graubard, BI. Infant feeding and childhood cancer. Lancet 1988;2:365-8.

13. Shu XO, Clemens J, Zheng W, Ying DM, Ji BT, Jin F. Infant breastfeeding and the risk of childhood lymphoma and leukaemia. Int J Epidemiol 1995;24:27-32

14. Petridou E, Trichopoulos D, Kalapothaki V, Pourtsidis A, Kogevinas M, Kalmanti M, et al. The risk profile of childhood leukaemia in Greece: a nationwide case-control study. Br J Cancer 1997;76:1241-7.

15. Schüz J, Kaletsch U, Meinert R, Kaatsch P, Michaelis J. Association of childhood leukaemia with factors related to the immune system. Br J Cancer 1999;80:585-90.

16. McKinney PA, Juszczak E, Findlay E, Smith K, Thomson CS. Preand perinatal risk factors for childhood leukaemia and other malignancies: a Scottish case control study. Br J Cancer 1999;80:1844 51 .

17. Dockerty JD, Skegg DC, Elwood JM, Herbison GP, Becroft DM, Lewis ME. Infections, vaccinations, and the risk of childhood leukaemia. Br J Cancer 1999;80:1483-9.

18. Rosenbaum PF, Buck GM, Brecher ML. Early child-care and preschool experiences and the risk of childhood acute lymphoblastic leukemia. Am J Epidemiol 2000;152:1136-44.

19. UK Childhood Cancer Study Investigators. Breastfeeding and childhood cancer. Br J Cancer 2001;85:1685-94.

20. Hardell L, Dreifaldt AC. Breast-feeding duration and risk of malignant diseases in childhood in Sweden. European J Clin Nutr 2001;55:179-85.

21. Kwan, ML. Breastfeeding and risk of childhood acute lymphoblastic leukemia [master's thesis]. Berkeley (CA): Division of Public Health Biology and Epidemiology, School of Public Health, Univ. of California, Berkeley; 2002.

22. Golding J, Paterson M, Kinlen LJ. Factors associated with childhood cancer in a national cohort study. Br J Cancer 1990;62:304-8.

23. Murray L, McCarron P, Bailie K, Middleton R, Davey Smith G, Dempsey S, et al. Association of early life factors and acute lymphoblastic leukaemia in childhood: historical cohort study. Br J Cancer 2002;86:356-61

24. Shu XO, Linet MS, Steinbuch M, Wen WQ, Buckley JD, Neglia JP, et al. Breast-feeding and risk of childhood acute leukemia. J Natl Cancer Inst 1999;91:1765-72.

25. Smulevich VB, Solionova LG, Belyakova SV. Parental occupation and other factors and cancer risk in children: I. Study methodology and non-occupational factors. Int J Cancer 1999;83:712-7.

26. Infante-Rivard C, Fortier I, Olson, E. Markers of infection, breastfeeding and childhood acute lymphoblastic leukaemia. Br J Cancer 2000;83:1559-64.

27. Bener A, Denic S, Galadari S. Longer breast-feeding and protection against childhood leukaemia and lymphomas. Eur J Cancer 2001;37:234-8.
28. Scott JA, Aitkin I, Binns CW, Aroni RA. Factors associated with the duration of breastfeeding amongst women in Perth, Australia. Acta Paediatr 1999;88:416-21.

29. Reinier KS. Methodological issues in epidemiologic studies of childhood leukemia: control selection and exposure assessment [dissertation]. Berkeley: Univ. of California at Berkeley; 2002.

30. Lancashire RJ, Sorahan T. Breastfeeding and childhood cancer risks: OSCC data. Br J Cancer 2003;88:1035-7.

31. Mathur GP, Gupta N, Mathur S, Gupta V, Pradhan S, Dwivedi JN, et al. Breastfeeding and childhood cancer. Indian Pediatr 1993;30:651-7.

32. Mwanda OW. Cancers in children younger than age 16 years in Kenya. East Afr Med J 1999;76:3-9.

33. Schwartzbaum JA, George SL, Pratt CB, Davis B. An exploratory study of environmental and medical factors potentially related to childhood cancer. Med Pediatr Oncol 1991;19:115-21.

34. Hartley AL, Birch JM, McKinney PA, Blair V, Teare MD, Carrette J, et al. The Inter-regional Epidemiological Study of Childhood Cancer (IRESCC): past medical history in children with cancer. J Epidemiol Community Health 1988;42:235-42.

35. McKinney PA, Cartwright RA, Saiu JM, Mann JR, Stiller CA, Draper GJ, et al. The Inter-regional Epidemiological Study of Childhood Cancer (IRESCC): a case control study of aetiological factors in leukaemia and lymphoma. Arch Dis Child 1987;62:279-87.

36. Perrillat F, Clavel J, Auclerc MF, Baruchel A, Leverger G, Nelken B, et al. Day-care, early common infections and childhood acute leukaemia: a multicentre French case-control study. Br J Cancer 2002;86:1064-9

37. van Steensel-Moll HA, Valkenburg HA, van Zanen GE. Childhood leukemia and infectious diseases in the first year of life: a registerbased case-control study. Am J Epidemiol 1986;124:590-4.

38. Kaatsch P, Kaletsch U, Meinert R, Miesner A, Hoisl M, Schüz J, et al. German case control study on childhood leukaemia: basic considerations, methodology and summary of the results. Klinische Padiatrie 1998;210:185-91

39. Davis MK. Breastfeeding and chronic disease in childhood and adolescence. Pediatr Clin North Am 2001;48:125-41, ix.

40. Ross JA, Potter JD, Robison LL. Infant leukemia, topoisomerase II inhibitors, and the MLL gene. J Natl Cancer Inst 1994;86:1678-80.

41. Greenland S. Quantitative methods in the review of epidemiologic literature. Epidemiol Rev 1987:9:1-30.

42. DerSimonian R, Laird N. Meta-analysis in clinical trials. Control Clin Trials 1986; 7:177-88.

43. Shore RE, Gardner MJ, Pannett, B. Ethylene oxide: an assessment of the epidemiological evidence on carcinogenicity. Br J Ind Med 1993;50:971-97.

44. Light RJ, Pillemer DB. Summing up: the science of reviewing research. Cambridge: Harvard University Press; 1984.

45. Begg CB, Mazumdar M. Operating characteristics of a rank correlation test for publication bias. Biometrics 1994;50:1088-101.

46. Weinstein HJ, Tarbell NJ. Leukemias and lymphomas of childhood. In: DeVita VT, Hellman S, Rosenberg SA, editors. Cancer: principles and practice of oncology. Vol. 2. 5th ed. New York: Lippincott Williams \& Wilkins; 1997. p. 2145-54

47. Martinez GA, Krieger FW. 1984 milk-feeding patterns in the United States. Pediatrics 1985;76:1004-8.

48. Li R, Ogden C, Ballew C, Gillespie C, Grummer-Strawn L. Prevalence of exclusive breastfeeding among US infants: the Third National Health and Nutrition Examination Survey (Phase II, 19911994). Am J Public Health 2002;92:1107-10.

49. Huttly SR, Barros FC, Victora CG, Beria JU, Vaughan JP. Do mothers overestimate breast feeding duration? an example of recall bias from a study in southern Brazil. Am J Epidemiol 1990;132:572-5.

50. Tuttle CR, Dewey KG. Potential cost savings for Medi-Cal, AFDC, Food Stamps, and WIC programs associated with increasing breastfeeding among low-income Hmong women in California. J Am Diet Assoc 1996;96:885-90.

51. Norris JM, Scott FW. A meta-analysis of infant diet and insulindependent diabetes mellitus: do biases play a role? Epidemiology 1996;7:87-92.

52. Slattery ML, Edwards SL, Caan BJ, Kerber RA, Potter JD. Response rates among control subjects in case-control studies. Ann Epidemiol $1995 ; 5: 245-9$ 